\title{
In vitro Quorum Quenching Activity of Eleusine indica Crude Ethanolic Extract against Pseudomonas aeruginosa and Serratia marcescens
}

\author{
Allan John R. Barcena, ${ }^{1}$ Eunice Maricar M. Baldovino, ${ }^{1}$ Justin Grace Bañez, ${ }^{1}$ \\ Czarina Ann B. Baptisma, ${ }^{1}$ Aldwin Matthew M. Baronda, ${ }^{1}$ Renren B. Barroga,${ }^{1}$ Jumela Mica Q. Bautista, ${ }^{1}$ \\ Gabriel Roberto G. Baybay, ${ }^{1}$ Rafael Mariano G. Baybay, ${ }^{1}$ Vibiene Norma C. Bernal, ${ }^{1}$ Katherine Adrielle R. Bersola, ${ }^{1}$ \\ Katrina Ysabelle T. Bolaños, ${ }^{1}$ Hans Joren L. Bondoc, ${ }^{1}$ Julius Ervin S. Buitizon, ${ }^{1}$ Alec Xavier D. Bukuhan, ${ }^{1}$ \\ John Patrick B. Bulaong, ${ }^{1}$ Jan Louise DC. Cabrera, ${ }^{1}$ Nikko H. Cabrestante, ${ }^{1}$ Gian Carlo M. Cabuco, ${ }^{1}$ \\ Jose Paciano B.T. Reyes, $\mathrm{MD}^{2}$ and Fresthel Monica M. Climacosa, MD, PhD ${ }^{3}$ \\ ${ }^{1}$ College of Medicine, University of the Philippines Manila \\ ${ }^{2}$ Department of Pharmacology and Toxicology, College of Medicine, University of the Philippines Manila \\ ${ }^{3}$ Department of Medical Microbiology, College of Public Health, University of the Philippines Manila
}

\begin{abstract}
Introduction. Nosocomial contaminants such as Pseudomonas aeruginosa and Serratia marcescens are increasingly developing resistance to many antibiotics. One of the promising alternatives that may complement, if not substitute, the use of antibiotics is quorum quenching, the process of interfering with chemical signals that mediate communication between microorganisms. Eleusine indica, a ubiquitous grass used traditionally to treat infections, has been shown to contain metabolites, such as fatty acid derivatives and p-coumaric acid, capable of quorum quenching. To date, there has been no study on the quorum quenching activity of $E$. indica.
\end{abstract}

Objectives. This study aimed to determine the in vitro activity of crude ethanolic extract of $E$. indica leaves against selected quorum-sensing regulated virulence factors of $P$. aeruginosa and $S$. marcescens.

Methodology. E. indica leaves were collected, washed, air-dried, and homogenized. Following ethanolic extraction and rotary evaporation, the extract was screened for antimicrobial activity through disk diffusion test and broth microdilution assay. The quorum quenching activity of the extract against $P$. aeruginosa was measured through swarming motility assay, while the activity against $S$. marcescens was measured through swarming motility and pigment inhibition assays. The quorum quenching assays were conducted in triplicates, and analysis of variance (ANOVA) was performed to identify differences among the treatment groups.

Results. Disk diffusion test revealed that no zones of inhibition formed against both P. aeruginosa and S. marcescens for varying concentrations of up to $200 \mathrm{mg} / \mathrm{mL}$ of the crude extract. Likewise, the MIC of the extract against both P. aeruginosa and S. marcescens was determined to be $>200 \mathrm{mg} / \mathrm{mL}$. However, it was shown that the extract, at $50 \mathrm{mg} / \mathrm{mL}$, has statistically significant activity $(\mathrm{p}<0.05)$ against the swarming motility of $P$. aeruginosa, and it is $71.6 \%$ as effective in reducing the swarming area of the bacteria compared to cinnamaldehyde. This was not observed when the extract was tested against the swarming motility of and pigment production by S. marcescens.

Conclusion. In this study, the quorum quenching activity of the crude ethanolic extract of $E$. indica leaves was found to be effective against $P$. aeruginosa but not against $S$. marcescens. The compounds that will be identified by further studies may conceivably be used as an adjunct therapy in P. aeruginosa infections and as coating agents in medical devices.

Key Words: Eleusine indica, Pseudomonas aeruginosa, quorum quenching, swarming motility, Serratia marcescens, prodigiosin

Corresponding author: Allan John R. Barcena

College of Medicine, University of the Philippines Manila

547 Pedro Gil Street, Ermita, Manila 1000, Philippines

Email: arbarcena1@up.edu.ph 


\section{INTRODUCTION}

The inappropriate use of antibiotics worldwide is accelerating the development of antimicrobial resistance in various microorganisms. With a growing number of antibiotics losing their efficacy, infections are becoming harder to treat, and the increasing number of infections due to resistant microorganisms has led to increased morbidity, mortality, length of hospitalization, and overall healthcare expenditure across the globe. ${ }^{1}$ In fact, among the most important focal points for the spread of resistance are hospitals where resistant microbes cause severe nosocomial infections. One of the promising alternatives that may complement, if not substitute, the use of antibiotics in treating infections is quorum quenching - the process of extinguishing the signals that mediate quorum sensing.

Quorum sensing is a chemically mediated type of communication among bacterial cells that allows them to function as a unit. ${ }^{2}$ The compounds that mediate this process are called autoinducers, and most Gram-negative bacteria utilize a specific class of compounds called acylhomoserine lactone (AHL) autoinducers. ${ }^{3}$ In an AHL system, the signal molecule is produced constitutively at low levels insufficient to trigger a single bacterium's receptors. However, when a bacterial population reaches a threshold density, the concentration of the signal can shift the equilibrium towards the binding of the signal to the receptor. The signal-receptor complex then acts as a transcription factor that promotes further production of the signal and expression of specific virulence factors such as swarming motility, pigment production, and biofilm formation, depending on bacterial density. ${ }^{4}$

By attenuating this communication system, inhibition of quorum sensing imposes lesser selective pressure for the development of resistance when compared to directly killing the pathogen. With antibiotics, especially when misused, a drug-resistant strain is given a survival advantage wherein it is allowed to proliferate without having to compete with nonresistant strains. In quorum sensing inhibition or quorum quenching, however, even if a bacterium eventually develops resistance against the inhibitor, it still has to compete with the whole bacterial population for survival. In addition, the majority of the bacterial population will be unable to produce the threshold signal to induce expression of the virulence factors by both non-resistant and resistant strains. ${ }^{5}$

As AHL autoinducers mediate quorum sensing in most Gram-negative microorganisms, the process of quorum sensing in Gram-negative pathogens, such as Pseudomonas aeruginosa and Serratia marcescens, may be disrupted by various quorum quenching mechanisms that interfere with the AHL system. These mechanisms include inhibition of AHL synthase expression, direct inhibition of AHL molecules, degradation of the AHL autoinducers, and utilization of synthetic analogs that mimic AHL molecules. ${ }^{6}$ Interestingly, a variety of plant-derived molecules and compounds, which include halogenated furanones, gamma-aminobutyric acid (GABA), pyrogallol, curcumin, ursolic acid, rosmarinic acid, fatty acid derivatives, phenolic compounds, flavanones, flavonoids, and flavonols, have been shown to inhibit quorum sensing in human pathogens through mechanisms previously mentioned. ${ }^{4,7}$ Eleusine indica, a ubiquitous grass, locally known as paragis, was reported to contain some of these quorum-quenching compounds. This grass has been used in many communities as a treatment for vaginal and urinary tract infections. A study by Penaloza et al. in 2018, which utilized mass spectrometry (MS) and nuclear magnetic resonance (NMR) spectroscopy, found that the aerial parts of $E$. indica contain fatty acid derivatives and p-coumaric acid. ${ }^{9}$ Several of these fatty acids have demonstrated activity against the swarming motility of $P$. aeruginosa in a study by Inoue et al. in 2008, while p-coumaric acid has demonstrated activity against Agrobacterium tumefaciens NTL4, Chromobacterium violaceum 5999, Pseudomonas chlororaphis in a study by Bodini et al. on soil bacteria in 2009..$^{10,11}$

To date, there has been no study on the efficacy of $E$. indica in combating bacterial infections through quorum sensing inhibition. This study aimed to determine the effects of crude ethanolic extract of $E$. indica leaves against the growth and production of quorum sensing-regulated virulence factors of $P$. aeruginosa and $S$. marcescens.

\section{METHODS}

\section{Plant collection and extraction}

$E$. indica leaves were collected from the University of the Philippines Diliman. Soil sample (1 kg) was also collected for heavy metal analysis. The leaves were thoroughly washed with distilled water and were air-dried for seven days. Following blender homogenization, the leaves were soaked in $95 \%$ ethanol at a $1: 2 \mathrm{w} / \mathrm{v}$ ratio for three days. The solution was then filtered using Whatman filter paper no. 4 into a round bottom flask. The filtrate was heated at $40^{\circ} \mathrm{C}$ at $100 \mathrm{rpm}$ using a rotary evaporator until the solvent has completely evaporated.

\section{Antimicrobial susceptibility testing}

To determine the susceptibility of $P$. aeruginosa and $S$. marcescens to the extract, disk diffusion test and broth microdilution assay were performed.

\section{Disk diffusion test}

Mueller-Hinton (MH) agar plates were inoculated evenly with the test microorganisms $-P$. aeruginosa and $S$. marcescens. Filter paper disks (6 $\mathrm{mm}$ in diameter) were loaded with $20 \mu \mathrm{L}$ of the different concentrations of the crude extract. Disks loaded with sterile water were used as the negative control, while disks loaded with $10 \mu \mathrm{g}$ of gentamicin were used as positive control following the standard protocol of the Clinical and Laboratory Standards, Institute (CLSI). The disks were then placed in the appropriate plate quadrants. Each setup was prepared in quintuplicates for 
each test microorganism, and the plates were incubated at $37^{\circ} \mathrm{C}$ for 24 hours.

\section{Broth microdilution assay}

On a sterile 96-well plate, $50 \mu \mathrm{L}$ of the $200 \mathrm{mg} /$ $\mathrm{mL}$ crude plant extract was pipetted into 3 columns for $P$. aeruginosa and 3 columns for $S$. marcescens. The first well in each column contained $200 \mathrm{mg} / \mathrm{mL}$ of the crude extract and each succeeding well down each column contained half of the previous well's concentration. The $8^{\text {th }}$ well contained only $1.56 \mathrm{mg} / \mathrm{mL}$ of crude extract. To each well, $40 \mu \mathrm{L}$ of Mueller-Hinton broth and $10 \mu \mathrm{L}$ of the bacterial suspension were added to obtain an organism concentration of $1 \times 10^{5}$ $\mathrm{CFU} / \mathrm{mL}$ per well. The positive control contained dilutions of gentamicin, while the negative control only contained $90 \mu \mathrm{L}$ of $\mathrm{MH}$ broth and $10 \mu \mathrm{L}$ of inoculum. The plate was incubated for 24 hours.

\section{Swarming motility assay for P. aeruginosa and S. marcescens}

Swarming motility media infused with the crude extract $(50 \mathrm{mg} / \mathrm{mL})$ was prepared and dispensed into Petri plates. Sterile distilled water was used as the negative control, and $50 \mathrm{mg} / \mathrm{mL}$ cinnamaldehyde was used as the positive control. After drying for 60 minutes, $5 \mu \mathrm{L}$ of bacteria suspended in buffered saline with an $\mathrm{OD}_{600}$ of $3.0\left(1.5 \times 10^{9} \mathrm{CFU} / \mathrm{mL}\right)$ was inoculated in the middle of each plate. Motility was observed after incubation for 24 hours. Using ImageJ, the surface area covered by the bacteria was determined. The experiment was performed in triplicates.

\section{Pigment inhibition assay for S. marcescens}

$S$. marcescens was inoculated into brain heart infusion (BHI) broth with $1 \%$ inoculum and either $50 \mathrm{mg} / \mathrm{mL}$ of the extract, sterile distilled water (negative control), or 50 $\mathrm{mg} / \mathrm{mL}$ cinnamaldehyde (positive control). After 24 hours of incubation, the samples were subjected to centrifugation ( $8,000 \mathrm{rpm} ; 5$ minutes) to extract prodigiosin, an antibiotic and pro-apoptotic pigment produced by $S$. marcescens. The supernatant was discarded, and the pellet was resuspended in acidified ethanol ( $4 \% 1 \mathrm{M} \mathrm{HCl}$ in ethanol). The remaining cell debris was removed by re-centrifugation, and the supernatant was transferred to a 96-well plate for measurement of absorbance at $534 \mathrm{~nm}$. Three replicates were prepared per test. A blank was prepared for each set-up to eliminate the effect of the extract's color on the absorbance values.

\section{Data collection and analysis}

Analysis of variance (ANOVA) was used to compare the different treatment groups. Simple ANOVA, followed by Tukey post-hoc testing, was performed for data that satisfied the Levene's test of homogeneity $(p<0.05)$. On the other hand, ANOVA-Welch test, followed by GamesHowell post-hoc testing, was performed for data which did not satisfy the test of homogeneity.

\section{RESULTS}

\section{Heavy metal testing}

The sample of the soil from which the plant samples were collected did not contain cadmium and nickel. On the other hand, the soil sample contained natural levels of arsenic $(0.568 \mathrm{mg} / \mathrm{kg})$ and lead $(37 \mathrm{mg} / \mathrm{kg})$. The natural level of arsenic in soils ranges from 1 to $40 \mathrm{mg} / \mathrm{kg}$, while the natural level of lead in soils ranges from 15 to $40 \mathrm{mg} / \mathrm{kg}$.

\section{Susceptibility testing}

The mean zone of inhibition of gentamicin is $26.1 \pm$ 0.65 for P. aeruginosa and $21.7 \pm 1.25$ for $S$. marcescens. Both values are within the susceptible range based on the standard protocol by the Clinical and Laboratory Standards, Institute (CLSI) and the American Society of Microbiology (ASM). No zones of inhibition formed for the negative control, and varying concentrations of the crude extract up to $200 \mathrm{mg} / \mathrm{mL}$. Similarly, we found that both $P$. aeruginosa and $S$. marcescens were susceptible only to the positive control upon performing the MIC test. The MIC of the positive control is less than the minimum tested concentration of gentamicin, which is $1.56 \mu \mathrm{g} / \mathrm{mL}$, for both $P$. aeruginosa and $S$. marcescens; this is consistent with the breakpoint value recommended by CLSI for gentamicin. On the other hand, no inhibition was observed for varying concentrations of the crude extract up to $200 \mathrm{mg} / \mathrm{mL}$. For the succeeding tests, one maximal dose $(50 \mathrm{mg} / \mathrm{mL})$ was used due to limitations in the amount of extract produced.

\section{Quorum sensing inhibition assay for $P$. aeruginosa}

The swarming surface area of $P$. aeruginosa was measured in $\mathrm{mm}^{2}$ using ImageJ. The mean and standard deviation of the surface area for the $50 \mathrm{mg} / \mathrm{mL}$ crude extract is 71.29 and 3.60 , respectively; for $50 \mathrm{mg} / \mathrm{mL}$ cinnamaldehyde, the mean and standard deviation are 14.49 and 0.56 , while for distilled water, 214.53 and 34.17. Since the data set did not satisfy the assumption for homogeneity confirmed by Levene's test for homogeneity $(\mathrm{p}<0.05)$, the Welch's $\mathrm{F}$ was used to compare the mean swarming surface area of $P$. aeruginosa exposed to $50 \mathrm{mg} / \mathrm{mL}$ crude extract, $50 \mathrm{mg} / \mathrm{mL}$ cinnamaldehyde, and distilled water; Games-Howell test was used for post-hoc analysis.

Figure 1 shows that the $50 \mathrm{mg} / \mathrm{mL}$ crude extract $(71.29 \pm 3.60)$ was able to inhibit the swarming motility of $P$. aeruginosa. There was a significant difference between all the groups, and posthoc comparisons using the GamesHowell test indicated that the swarming surface area of the cinnamaldehyde-exposed $P$. aeruginosa is significantly the smallest $(14.49 \pm 0.56)$ followed by the $50 \mathrm{mg} / \mathrm{mL}$ crude extract $(71.29 \pm 3.60)$, and distilled water had the highest surface area $(214.53 \pm 34.17)$. Hence, the $50 \mathrm{mg} / \mathrm{mL}$ crude extract has quorum quenching activity against the swarming motility of $P$. aeruginosa, although less compared to the positive control. The positive control caused a $93.25 \%$ 
reduction in the swarming area versus negative control, while the crude extract at $50 \mathrm{mg} / \mathrm{mL}$ caused a $66.77 \%$ reduction. Compared to the positive control, the extract at $50 \mathrm{mg} / \mathrm{mL}$ is $71.6 \%$ as effective in reducing the swarming area of $P$ aeruginosa.

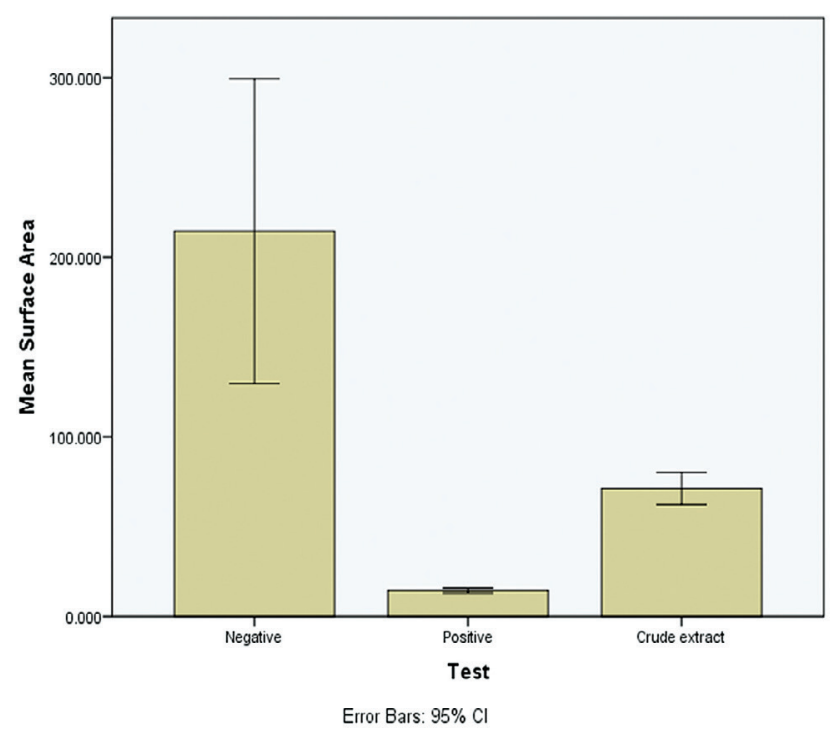

Figure 1. Mean surface area of P. aeruginosa swarming motility. The surface area covered by cinnamaldehydeexposed $P$. aeruginosa is the smallest (14.49 \pm 0.56 ), followed by the $50 \mathrm{mg} / \mathrm{mL}$ crude ethanolic extract (mean $=71.29 \pm 3.60)$ and then distilled water. (214.53 \pm 34.17$)$.

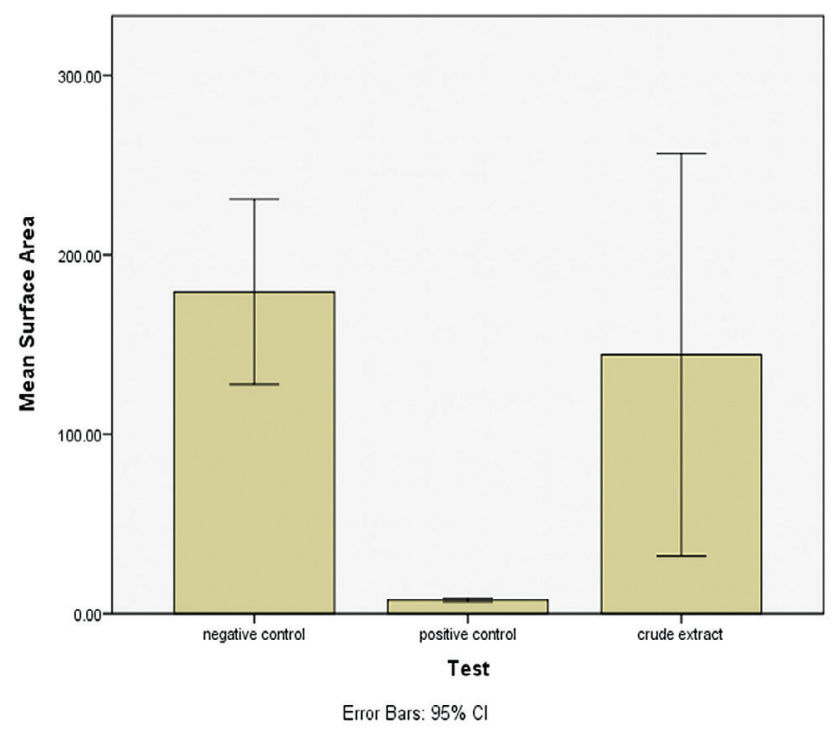

Figure 2. Mean surface area of $S$. marcescens swarming motility. The surface area of the cinnamaldehydeexposed S. marcescens is the smallest $(7.57 \pm 0.38)$ followed by the $50 \mathrm{mg} / \mathrm{mL}$ crude extract (144.38 \pm 45.18 ) and distilled water (179.42 \pm 20.77 ).
Quorum sensing inhibition assays for S. marcescens

Figure 2 shows that the mean swarming surface areas covered by $S$. marcescens exposed to $50 \mathrm{mg} / \mathrm{mL}$ crude extract, $50 \mathrm{mg} / \mathrm{mL}$ cinnamaldehyde, and distilled water, are 144.38, 7.57 , and 179.42 , respectively. The outcome variable was found to be normally distributed and the assumption for equal variances was fulfilled based upon the result of the Levene's test. Posthoc comparisons using Tukey HSD test revealed that there is a significant difference between positive control $(7.57 \pm 0.38)$ and the other two groups, but no significant difference between negative control $(179.42 \pm 20.77)$ and the $50 \mathrm{mg} / \mathrm{mL}$ crude extract $(144.38 \pm 45.18)$. Thus, the crude extract at $50 \mathrm{mg} / \mathrm{mL}$ had no quorum quenching activity against the swarming motility of $S$. marcescens.

The inhibition of prodigiosin production by $S$. marcescens was determined by spectrophotometry at $534 \mathrm{~nm}$ where a higher absorbance indicates the presence of a greater amount of prodigiosin. The mean absorbance measurements for 50 $\mathrm{mg} / \mathrm{mL}$ crude extract, $50 \mathrm{mg} / \mathrm{mL}$ cinnamaldehyde, and distilled water are $0.23,0.05$, and 0.19 , respectively. Levene's test revealed that the groups do not assume equal variances $(p<0.05)$, hence, Welch's $F$ was used to compare the mean absorbance values for each treatment. Figure 3 shows that there is a significant difference between positive control and the other two groups, but no significant difference between negative control and extract. Posthoc analysis using the Games-Howell test revealed that cinnamaldehyde $(0.05 \pm$ 0.002 ) is significantly different from the $50 \mathrm{mg} / \mathrm{mL}$ crude extract and distilled water. However, there is no significant difference between the $50 \mathrm{mg} / \mathrm{mL}$ crude extract $(0.23 \pm$ $0.02)$ and distilled water $(0.19 \pm 0.03)$. Therefore, the crude

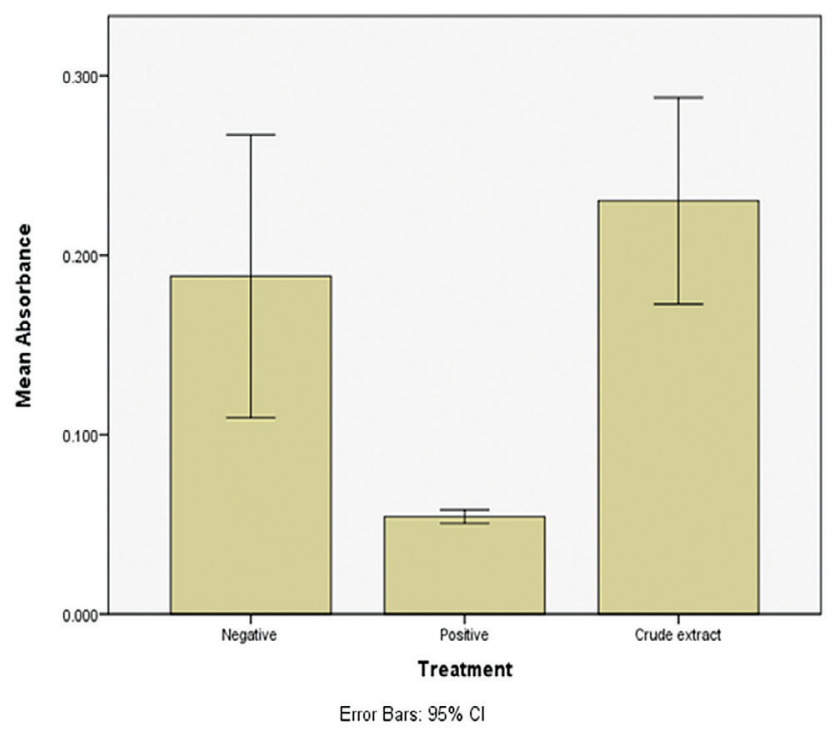

Figure 3. Pigment inhibition assay. Cinnamaldehyde has the lowest mean spectrophotometric absorbance value at $534 \mathrm{~nm}(0.054 \pm 0.002)$, followed by distilled water $(0.19 \pm 0.03)$, then the crude extract, which has the highest absorbance value $(0.23 \pm 0.02)$. 
extract at $50 \mathrm{mg} / \mathrm{mL}$ did not inhibit the quorum sensingdependent production of prodigiosin by $S$. marcescens.

\section{DISCUSSION}

\section{Susceptibility testing}

The results of the susceptibility tests show that the crude extract has no direct antimicrobial activity against $P$. aeruginosa and S. marcescens in all concentrations (up to 200 $\mathrm{mg} / \mathrm{mL}$ ) tested. The antimicrobial effect of $E$. indica on $P$. aeruginosa has been reported in the literature, but this is the first study to explore the antimicrobial effect of $E$. indica on $S$. marcescens. While the results appear to contradict the findings of previous studies on the antimicrobial activity of $E$. indica against $P$. aeruginosa, it is important to note that these studies have used different extraction methods and solvents. For example, the study by Al-Zubairi et al. in 2011 reported that $E$. indica hexane, dichloromethane, and ethyl acetate extracts have significant activity against $P$. aeruginosa. ${ }^{12}$ Another study by Alaekwe et al. in 2015, which tested the aerial parts of $E$. indica and found that the extracts exhibited antibacterial activity on different species of bacteria, including $P$. aeruginosa, has used chloroform and methanol as extraction solvents. ${ }^{13}$ Thus, the use of ethanol as a solvent in this study may have affected the antimicrobial activity of the extract. Although it appears to be an unsuitable solvent for the extraction of antimicrobial compounds, it allows for the separation of compounds that may be tested for their quorum quenching activity.

\section{Quorum sensing inhibition assay against $P$. aeruginosa}

It was shown that the extract (at $50 \mathrm{mg} / \mathrm{mL}$ ) has statistically significant activity $(\mathrm{p}<0.05)$ against the swarming motility of $P$. aeruginosa. Swarming, defined as movement across a semisolid medium, is important in the spread of infection and evasion of the host response. It is highly dependent on the cooperative production of proteases and surfactants, and it is easily measured through swarming motility assays.

The swarming motility of $P$. aeruginosa is controlled by a multi-layered quorum sensing network consisting of at least four interconnected systems-namely Las, Rhl, Iqs, and Pqs. The Las system is responsible for the production of elastases and proteases that are important in the breakdown of nutritional substrates and movement of bacterial cells across protein-rich tissues; this requires LasI synthase to produce $\mathrm{N}$-(3-oxododecanoyl)-L-homoserine lactone autoinducers which activate the LasR transcription factor. The Rhl system, on the other hand, is responsible for the production of rhamnolipids, the biosurfactants necessary for swarming and translocation of $P$. aeruginosa in semisolid surfaces; this system involves the Rhll synthase that produces $N$-butyryl-L-homoserine lactone autoinducers required by RhlR transcriptional regulators. The Las and
Rhl systems work in synchrony with the other systemsIqs (integrated QS signal) and Pqs (Pseudomonas quinolone signal) - to produce important virulence factors, which not only include swarming motility but also biofilm formation and antibiotic production. ${ }^{14}$

Due to the dependence of quorum sensing systems on AHL autoinducers, direct inactivation of autoinducers or blockage of autoinducer receptors may ultimately lead to quorum sensing inhibition. AHL analogs, including holoACP,L/D-S-adenosylhomocysteine, sinefungin, and butyrylS-adenosylmethionine, have been found to interfere with AHL production and, consequently, quorum sensing. ${ }^{15}$ Even molecules that have retained only a part of the cognate AHL molecule have been implicated in significantly lowering the LasR-controlled expression of important effector proteins. Several compounds that can act as quorum inhibitors have been isolated from fungi and plants, and the ability of the crude extract at $50 \mathrm{mg} / \mathrm{mL}$ to interfere with the swarming motility of $P$. aeruginosa suggests the presence of quorum sensing inhibitors in E. indica leaves as well.

Astudy by Penaloza et al.in 2018using NMRspectroscopy reported that the aerial parts of E. indica contained four specific compounds-flavonoids schaftoside, isoschaftoside, vitexin, and $p$-coumaric acid. ${ }^{9}$ They have also detected 29 unknown compounds which they grouped into fatty acid derivatives, amino acids, carbohydrates, and aromatics. ${ }^{9}$ Among all these components, flavonoids, p-coumaric acid, and fatty acid derivatives have the potential to promote the inhibition of the $P$. aeruginosa quorum-sensing network. A few plants, such as Centella asiatica L. and Psidium guajava $L$., which have been shown to inhibit the swarming motility of $P$. aeruginosa in a dose-dependent manner, have been reported to contain rich amounts of flavonoids, including kaempferol, quercetin, apigenin, rutin, and naringin. ${ }^{16}$ While the aforementioned flavonoids have not yet been identified to be present in $E$. indica, it is postulated that other flavonoids which are known to be present in E. indica also have to potential to inhibit quorum sensing. On the other hand, $\mathrm{p}$-coumaric acid has demonstrated quorum quenching activity against Agrobacterium tumefaciens NTL4, Chromobacterium violaceum 5999, and Pseudomonas chlororaphis in a study by Bodini et al. on soil bacteria in 2009. ${ }^{11}$ It is postulated to inhibit biofilm formation and swarming motility in selected bacteria by decreasing the expression of promoters of genes important for biosurfactant expression. Fatty acids may also account for the quorum sensing inhibition against $P$. aeruginosa. Inoue et al. showed that branched-chain fatty acids and unsaturated fatty acids, such as anteiso-C15:0, iso-C15:0, anteiso-C17:0, iso-C17:0, oleic acid, and vaccenic acid, inhibited the swarming motility of $P$. aeruginos ${ }^{10}{ }^{10}$ The inhibitory activity seemed to correspond to the length of the chain with a length greater than fourteen carbons exhibiting more suppressive activity. ${ }^{10}$

Contamination with specific heavy metals, such as cadmium and nickel, may also produce quorum quenching 
activity. However, the results of the heavy metal testing showed that the sample of soil from which the plant samples were collected did not contain cadmium and nickel. On the other hand, the soil sample contained natural levels of arsenic and lead. There are no reports in the literature that arsenic and lead can act as quorum sensing inhibitors.

\section{Quorum sensing inhibition assay against $S$. marcescens}

Swarming motility is also a quorum sensing-regulated virulence factor of $S$. marcescens. The quorum sensing network of $S$. marcescens has three key regulators-namely Swr, Sma, and Spn; both the Swr and Sma systems are the main controllers of swarming motility. The Swr system primarily regulates the production of serrawettin, proteases, and S-layer proteins, while the Sma system regulates the production of caseinases and chitinases. The most notable surfactant molecule involved in the swarming motility of $S$. marcescens is serrawettin, an extracellular lipopeptide that reduces surface tension. Studies involving surfactant-defective phenotypes showed that knockouts had reduced swarming capability, which was quickly restored by supplementing the medium with serrawettin. ${ }^{17}$

Unlike in the case of P. aeruginosa, the crude extract at $50 \mathrm{mg} / \mathrm{mL}$ has no significant activity against the swarming motility of $S$. marcescens. The swarming motility of $S$. marcescens is less studied, and some of the compounds that have been shown to inhibit the swarming motility of $P$. aeruginosa, such as flavonoids and fatty acids, are not known to inhibit the swarming motility of $S$. marcescens. However, a study in 2014 showed that biofilm formation, a phenotype closely related to swarming motility, by $S$. marcescens can be inhibited by a phenolic compound-phenol, 2,4-bis(1,1dimethylethyl) - produced by Vibrio alginolyticus. ${ }^{18}$ This just shows that quorum sensing is specific and that inhibitors of quorum sensing in one species may not work for other bacteria. Other known inhibitors of swarming motility in Serratia species include halogenated furanone, $\mathrm{Cn}-\mathrm{CPA}$, and Anethum graveolens methanolic extract. Halogenated furanone, produced by Delisea pulchra was observed to have inhibitory effects on $S$. liquefaciens swarming motility. ${ }^{19}$ $\mathrm{Cn}-\mathrm{CPA}$, which interferes with quorum sensing-regulated virulence factors of $S$. marcescens, was found to inhibit the swarming motility and biofilm formation of $S$. marcescens; it was also found to be more effective than the halogenated furanone. ${ }^{19} A$. graveolens methanolic extract, on the other hand, has been shown to downregulate quorum sensingregulated expression of transcription of factors involved in adhesion and motility. ${ }^{20}$

While the motility of both P. aeruginosa and S. marcescens has an established association with their virulence, the mechanisms involved in each species' swarming motility are different. The swarming cell of $P$. aeruginosa is elongated, some possessing two polar flagella with or without pili. ${ }^{14}$ S. marcescens, on the other hand, has an elongated, multinucleated, aseptate, and hyper-flagellated swarming cell. ${ }^{17}$ Thus, inhibition of flagellar movement may be a more important target in the swarming motility of $S$. marcescens. A study by Devi et al. in 2018 shows that B. subtilis $\mathrm{R}-18 \mathrm{PE}$ extract inhibited swimming and swarming in $S$. marcescens by reducing flagella-mediated movements. ${ }^{21}$ The biosurfactants that $P$. aeruginosa and $S$. marcescens produce are also different, specifically rhamnolipid for $P$. aeruginosa and serrawettin for S. marcescens. ${ }^{14,17}$

Prodigiosin production is also a quorum sensingregulated virulence factor of $S$. marcescens. Of the three key regulators of the quorum sensing network of $S$. marcescens, the Spn system is the one primarily involved in regulating the production of prodigiosin in addition to nuclease production. ${ }^{17}$ Similar to swarming motility, the crude extract at $50 \mathrm{mg} / \mathrm{mL}$ has no significant activity against the prodigiosin production of $S$. marcescens. The prodigiosin production of $S$. marcescens is also less studied, and some of the compounds that have been shown to inhibit the swarming motility of $P$. aeruginosa, such as flavonoids, phenolic compounds, and fatty acids, are not known to inhibit prodigiosin production of S. marcescens. However, it has been shown that a few known compounds may inhibit the production of prodigiosin in $S$. marcescens. For example, a study by Morohoshi et al. found that $\mathrm{N}$-nonanoyl-cyclopentylamide (C9-CPA) exhibited a strong inhibitory effect on prodigiosin production by $S$. marcescens. ${ }^{19}$ Similarly, this confirms that inhibitors of quorum sensing in $P$. aeruginosa may not work for other bacteria, such as S. marcescens.

\section{CONCLUSION}

Since the discovery of quorum sensing in many pathogenic bacteria, quorum quenching mechanisms have been explored as a promising approach to combat the global health problem of antibiotic resistance and to develop novel medicines and combination therapies. In this research, the quorum quenching activity of $E$. indica at a concentration of $50 \mu \mathrm{g} / \mathrm{mL}$ was found to be effective against the swarming motility $P$. aeruginosa but not against the swarming motility and prodigiosin production of $S$. marcescens. With this, usage of $E$. indica quorum quenching activity offers a promising addition in treating $P$. aeruginosa-associated infections in which quorum sensing plays a significant role, such as microbial keratitis and cystic fibrosis opportunistic infection..$^{22}$ However, the application of E. indica quorum quenching activity against $S$. marcescens remains unproven. Nonetheless, E. indica could still be explored as an adjunct therapy in nosocomial infections, which are most commonly caused by $P$. aeruginosa, especially with the rapid development of resistance by $P$. aeruginosa to multiple classes of antibiotics that are currently used in most hospitals around the world.

Aside from having direct therapeutic applications, quorum quenching compounds that will be isolated from $E$. indica ethanolic extract may also be applied on medical 
devices such as dressings and catheters to prevent nosocomial infections. We look forward to more studies on the quorum quenching activity of $E$. indica ethanolic extract using other concentrations to determine the presence of a dose-response relationship. We also look forward to studies on identifying the specific mechanisms involved in the inhibition of $P$. aeruginosa swarming motility following isolation for possible large-scale synthetic production, and on exploring the effect of $E$. indica ethanolic extract on other pathogens.

\section{Statement of Authorship}

All authors have approved the final version submitted.

\section{Author Disclosure}

All authors declared no conflicts of interest.

\section{Funding Source}

This study was funded by the Department of Pharmacology and Toxicology, College of Medicine, University of the Philippines Manila.

\section{REFERENCES}

1. Centers for Disease Control and Prevention. Antibiotic or Antimicrobial Resistance: About Antimicrobial Resistance [Internet]. 2018 [cited 2019 Aug]. Available from https://www.cdc.gov/drug resistance/about.html.

2. Waters CM,Bassler BL. Quorum Sensing: Cell-to-cell Communication in Bacteria. Annual Review of Cell and Developmental Biology. 2005;21:319-46.

3. Gonzalez JE, Keshavan ND. Messing with Bacterial Quorum Sensing. Microbiology and Molecular Biology Reviews. 2006;70(4):859-75.

4. Nazzaro F, Fratianni F, Coppola R. Quorum Sensing and Phytochemicals. International Journal of Molecular Sciences. 2013; 14(6):12607-19.

5. Koh C, Sam C, Yin W, Tan L, Krishnan T, Chong Y, et al. Plant-derived Natural Products as Sources of Anti-quorum Sensing Compounds. Sensors. 2013;13(5):6217-228.

6. Kalia V. Quorum Sensing Inhibitors: An Overview. Biotechnology Advances. 2013;31(2):224-45.

7. Adonizio AL, Downum K, Bennett BC, Mathee K. Antiquorum Sensing Activity of Medicinal Plants in Southern Florida. Journal of Ethnopharmacology. 2006;105:427-35.

8. Vattem DA, Mihalik K, Crixell SH, McLean RJ. Dietary Phytochemicals as Quorum Sensing Inhibitors. Fitoterapia. 2007; 78: 302-10.
9. Peñaloza EMC, Casanova LM, Leal ICR, De Aguiar PF, Costa SS. Metabolite Fingerprinting and Profiling of the Medicinal Grass Eleusine indica based on HPLC-DAD, UPLC-DAD-MS/MS and NMR Analyses. Journal of the Brazilian Chemical Society. 2018; 29(12):2522-34.

10. Inoue T, Shingaki R, Fukui K. Inhibition of Swarming Motility of Pseudomonas aeruginosa by Branched-chain Fatty Acids. FEMS Microbiology Letters. 2008;281(1):81-6.

11. Bodini SF, Manfredini S, Epp M, Valentini S, Santori F. Quorum Sensing Inhibition Activity of Garlic Extract and p-coumaric Acid. Letters in Applied Microbiology. 2009;49(5):551-5.

12. Al-Zubairi A, Abdul AB, Abdelwahab SI, Peng CY, Mohan S, Elhassan MM. Eleucine indica Possesses Antioxidant, Antibacterial and Cytotoxic Properties. Evidence-Based Complementary and Alternative Medicine. 2011;2011:1-6.

13. Alaekwe IO, Ajiwe, VIE, Ajiwe, AC, Aningo, GN. Phytochemical and Anti-microbial Screening of the Aerial Parts of Eleusine indica. International Journal of Pure and Applied Bioscience. 2015;3(1): 257-64.

14. Lee J, Zhang L. The Hierarchy Quorum Sensing Network in Pseudomonas aeruginosa. Protein Cell. 2015;6(1):26-41.

15. Parsek MR, Val DL, Hanzelka BL, Cronan, JE, Greenberg EP. Acyl Homoserine-lactone Quorum-sensing Signal Generation. Proceedings of the National Academy of Sciences of the United States of America. 1999;96(8):4360-5.

16. Vasavi HS, Arun AB, Rekha, PD. Anti-quorum Sensing Activity of Psidium guajava L. Flavonoids Against Chromobacterium violaceum and Pseudomonas aeruginosa PAO1. Microbiology and Immunology. 2014;58(5):286-93.

17. Van Houdt R, Givskov M, Michiels CW. Quorum Sensing in Serratia. FEMS Microbiology Reviews. 2008;31:407-24.

18. Padmavathi AR, Abinaya B, Pandian SK. Phenol, 2,4-bis(1,1dimethylethyl) of Marine Bacterial Origin Inhibits Quorum Sensing Mediated Biofilm Formation in the Uropathogen Serratia marcescens. Biofouling. 2014;30(9):1111-22.

19. Morohoshi T, Shiono T, Takidouchi K, Kato M, Kato N, Kato J, et al. Inhibition of Quorum Sensing in Serratia marcescens AS-1 by Synthetic Analogs of N-Acylhomoserine Lactone. Applied and Environmental Microbiology. 2007;73(20):6339-44.

20. Salini R, Pandian SK. Interference of quorum sensing in urinary pathogen Serratia marcescens by Anethum graveolens. Pathogens and Disease. 2015;73(6).

21. Devi KR, Srinivasan S, Ravi AV. Inhibition of Quorum Sensingmediated Virulence in Serratia marcescens by Bacillus subtilis R-18. Microbial Pathogenesis. 2018;120:166-75.

22. Willcox MDP, Zhu H, Conibear TC, Hume EB, Givskov M, Kjelleberg S, Rice SA. Role of Quorum Sensing by Pseudomonas aeruginosa in Microbial Keratitis and Cystic Fibrosis. Microbiology. 2008;154:2184-94. 